\title{
A Rapid, Empirical Method for Detection and Estimation of Outlier Frames in Particle Imaging Velocimetry Data Using Proper Orthogonal Decomposition
}

\author{
J. Higham, W. Brevis, C. J. Keylock \\ Sheffield Fluid Mechanics Group and Department of Civil \& Structural Engineering, University of Sheffield.
}

\begin{abstract}
This paper develops a method for detection and removal of outlier images from digital Particle Image Velocimetry data using Proper Orthogonal De-composition (POD). The outlier is isolated in the leading POD modes, removed and a replacement value reestimated. The method is used to estimate and replace whole images within the sequence. This is particularly useful, if a single PIV image is suddenly heavily contaminated with background noise, or to estimate a dropped frame within a sequence. The technique is tested on a synthetic dataset that permits the effective acquisition frequency to be varied systematically, before application to flow field frames obtained from a large-eddy simulation. As expected, outlier re-estimation becomes more difficult when the integral time scale for the flow is long relative to the sampling period. However, the method provides a systematic improvement in predicting frames compared to interpolating from neighbouring frames.
\end{abstract}

Keywords Image Processing; Outlier Detection; Proper Orthogonal Decomposition; Particle Imaging Velocimetry

\section{INTRODUCTION}

Obtaining high quality experimental data for turbulent flows is a difficult task. Instrument noise, and seeding variation between frames can result in outlier data being generated at particular time instants. These problems are discussed for the case Particle Image Velocimetry (PIV) by [1] Outliers are defined in this paper to be singular frames within a data series that do not correlate to the value anticipated based on the properties of the neighbouring data. PIV is a nonintrusive measurement technique, typically employed for detailed laboratory investigations of fluid mechanical processes [1]. It is based on the acquisition of images of the movement of tracer particles (normally neutrally buoyant particles). The temporal acquisition of these images should be such that the correlation between particles patterns between two sequential images is high enough to estimate their average displacement typically using Fourier methods to estimate the velocity vectors.

Because of unexpected experimental issues and flow nonlinearity, data obtained at particular times may be significantly less well seeded than at other times, despite the best attempts to minimise experimental error. A lack of seeding, and a low temporal acquisition may lead to the decorrelations of pairs of images and consequently outlier frames. Naturally, outlier detection and removal has been an area of some research interest. However, the majority of work has focused on the treatment of spurious outlier vectors, rather than the statistical properties of the whole frame $[5,7]$.

\section{POD}

Proper Orthogonal Decomposition (POD) is a commonly used, linear method, for the extraction and analysis of turbulent structures [2, 3] and for forming low order representations of the dynamics of fluid flows for modelling and control purposes [8]. POD extracts energy relevant structures from a stochastic, statistically steady-state turbulent field, within a finite time domain, and orders them by their contribution to the total variance or, equivalently, by their specific energy (Kostas et al., 2005). We can define the singular value decomposition in terms of the eigenvalues, $\lambda_{n}$, as well as spatial $\phi_{n}(x)$, and temporal $\alpha_{n}(t)$ modes: as:

$$
\boldsymbol{v}=\sum_{n-1}^{N} \lambda_{n} \phi_{n}(x) \alpha_{n}(t)
$$

where $t$ is time and $n=1, \ldots, N$ number of modes, but is structured in terms of the descending rank order of the $\lambda_{n}$.

\section{MethOD}

Our method works by decomposing an ensemble of frames containing an outlier frame into three products using the POD method, producing sets of eigenvalues $\lambda_{n}$; eigenvectors $\phi_{n}$ and coefficients $\alpha_{n}$. The $\lambda_{n}$ give the energy contribution per mode, the eigenvectors, $\phi_{n}$ the orientation of the mode in the variable space and these eigenfunctions are related temporally using the coefficients $\alpha \mathrm{n}$. It is these coefficients $\alpha_{n} \alpha_{n}$ which our method exploits to estimate outlier frames. If there is an outlier frame within the time domain, a spike is depicted in the $\alpha_{n}$ values. As the "outlier" frames can be seen as a spike within the $\alpha_{n}$, it is possible to detect them quite easily. By removing and replacing this spike, it is possible to re-estimate the outlier frame using an interpolation method. Inverting the POD with the cleaned data permits the ensemble of frames to be reconstructed with the outlier replaced.

\section{RESULTS}

For this study two cases were selected the first being the "Lena Söderberg" image, which was circularly shifted to simulate the passive advection of a non-diffusive tracer distributed spatially in a complex fashion. The increment steps in the circular rotation were varied to simulate different sampling frequencies. The second dataset was a subset of Large Eddy Simulation (LES) of turbulent flow through a groyne field [4]. For both cases a sample length of 100 frames were used, of which frame 30 was replaced with a Gaussian white noise with a magnitude equal to that of the average of all of the frame minus the missing frame. Our 
method is compared to a linear interpolation method defined as $\left(V_{s(n-1)}+V_{s(n+1)}\right) / 2$ where $V_{s(n)}$ is the "outlier" frame, in the termed spurious data.

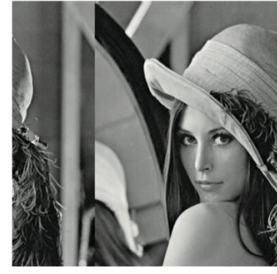

(a)

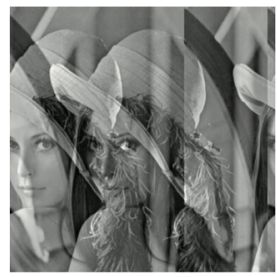

(c)

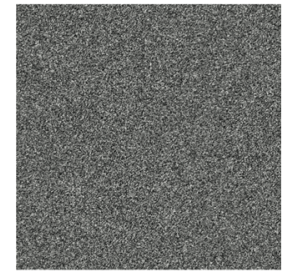

(b)

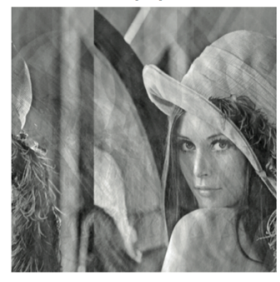

(d)
Figure 1. Lena data: (a). Shifted original image; (b). Noise added over image; (c). Linearly estimated image; (d). Our method.

It can be seen visually that our method has managed to produce an image that visually represents that of the original image better than the linear interpolation method. Fig. 1d has a Root-Mean Square Error (RMSE) of $21 \%$ defined as the RMSE normalised by the RMS of the original image. The error in Fig. 1 (c) is much greater at $58 \%$.

The error from our method in Fig. 1 (d) is $24 \%$, which is an improvement on the $31 \%$ error for the linear interpolated method. More importantly, our method has retained structures that were lost using linear interpolation. These are marked in green in Fig. $2 \mathrm{~d}$ and can also be seen in the second Lena face in Fig. 1(c).

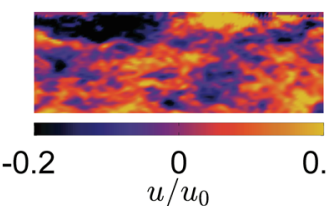

(a)

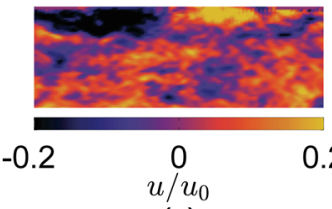

(c)

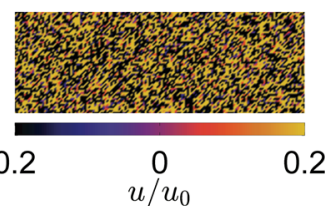

(b)

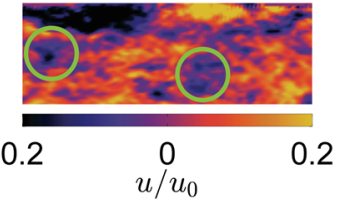

(d)
Figure 2. Groyne flow image: (a). Original Image; (b). Noise added over image; (c). Linearly estimated image; (d). Our method.

\section{CONCLUSIONS}

Our work so far indicates that our method offers a regular increase in accuracy to that of linear interpolation. Such a tool could be used in a variety of experimental situations where a hardware or experimental fault has led to an "outlier" or missing frame. Although the method does not offer a perfect solution, it does offer a means to increase the accuracy of prediction of an "outlier" frame.

\section{REFERENCES}

1. Adrian R, Westerweel J. Particle Image Velocimetry: Cambridge University Press; 2011.

2. Aubry N. On the Hidden Beauty of the Proper Orthogonal Decomposition. Theoretical and Computational Fluid Dynamics 2. 1991; no. 5-6: p. 339-52.

3. Berkooz G, Holmes P, Lumley aJL. The Proper Orthogonal Decomposition in the Analysis of Turbulent Flows. Annual Review Fluid Mechanics 25. 1993; no.1: p. 539-75.

4. Brevis W, M. GV, Y. N. Experimental and Large Eddy Simulation Study of the Flow Developed by a Sequence of Lateral Obstacles. Env. Fluid Mechanics. 2014.

5. Duncan J, D. D, J. H, M. G. Universal Outlier Detection for Particle Image Velocimetry (PIV) and Particle Tracking Velocimetry (PTV) Data. Meas. Sci. Tech. 21. 2010; no.5.

6. Kostas J, j. S, M. C. A Comparison between Snapshot POD Analysis of PIV Velocity and Vorticity Data. Exp. Fluids 38. 2005; no.2: p. 146-60.

7. Lumley J, P. B. Control of Turbulence. Ann. Rev. Fluid Mech. 30. 1998;: p. 311-27.

8. Westerweel J, F. S. Universal Outlier Detection for PIV Data. Exp. Fluids 39. 2005; no.6: p. 1096-1100. 